\title{
Desempenho agronômico de mucuna-verde em diferentes arranjos espaciais
}

\author{
Aijânio Gomes de Brito Silva(1), José Guilherme Marinho Guerra(2), Murilo Gonçalves Junior(1), \\ Janaína Ribeiro Costa ${ }^{(2)}$, José Antonio Azevedo Espíndola(2) e Ednaldo da Silva Araújo ${ }^{(2)}$
}

(1)Universidade Federal Rural do Rio de Janeiro, BR 465, Km 7, CEP 23890-000 Seropédica, RJ. E-mail: aijanio@yahoo.com.br,
murilojunior3@yahoo.com.br (2)Embrapa Agrobiologia, BR 465, Km 7, CEP 23890-000 Seropédica, RJ. E-mail: gmguerra@cnpab.embrapa.br,
janaina@cnpab.embrapa.br, jose@cnpab.embrapa.br, ednaldo@cnpab.embrapa

Resumo - O objetivo deste trabalho foi avaliar o efeito de diferentes arranjos espaciais no desempenho agronômico de mucuna-verde (Mucuna pruriens var. utilis). O experimento consistiu de oito tratamentos, dispostos em delineamento de blocos ao acaso em arranjo fatorial $2 \times 4$, com quatro repetições. Os tratamentos foram semeadura da mucuna-verde, em dois espaçamentos entre sulcos de plantio $(0,5$ e $1,0 \mathrm{~m})$ e quatro densidades de plantas $\left(2,4,8\right.$ e 16 plantas $\left.\mathrm{m}^{-1}\right)$. Determinaram-se as taxas de cobertura do solo e de fixação biológica de nitrogênio (FBN) e a produção de matéria seca e a quantidade de $\mathrm{N}$ acumulado na parte aérea. A cobertura total do solo ocorreu aos 50 dias após a semeadura, na densidade de 16 plantas $\mathrm{m}^{-1}$ e espaçamento de $0,5 \mathrm{~m}$ entre sulcos de plantio. A combinação da densidade de 16 plantas $\mathrm{m}^{-1}$ com o espaçamento de $1,0 \mathrm{~m}$ entre sulcos proporcionou a maior produção de matéria seca e quantidade de $\mathrm{N}$ acumulado na parte aérea das plantas. Independentemente do arranjo espacial das plantas, a estimativa da FBN nesta espécie mostra que cerca de $70 \%$ do $\mathrm{N}$ presente na parte aérea é derivado da atmosfera.

Termos para indexação: Mucuna pruriens var. utilis, adubo verde, fixação biológica de nitrogênio.

\section{Agronomic performance of velvet bean at different spatial arrangement}

\begin{abstract}
The objective of this work was to evaluate the effect of different plant spatial arrangements on agronomic performance of velvet-bean (Mucuna pruriens var. utilis). The experiment was performed with eight treatments, distributed in a randomized complete block design in a $2 \times 4$ factorial arrangement, with four replicates. The treatments were velvet bean sowing at two spacings between furrows $(0.5$ and $1.0 \mathrm{~m})$ and four plant densities $\left(2,4,8\right.$ and 16 plants $\left.\mathrm{m}^{-1}\right)$. Determinations were made for the soil covering and biological nitrogen fixation (BNF) rates, and for the dry matter yield and $\mathrm{N}$ accumulation in the plant shoots. Total soil cover was accomplished at 50 days after sowing at 16 plants $\mathrm{m}^{-1}$ density and $0.5 \mathrm{~m}$ spacing between furrows. The combination of 16 plants $\mathrm{m}^{-1}$ density with the $1.0 \mathrm{~m}$ spacing between furrows provided the greatest dry matter yield and accumulated most $\mathrm{N}$ in the plant shoots. Irrespective of the plant spatial arrangement, the estimation of BNF in this species shows that about $70 \% \mathrm{~N}$ present in the shoot is derived from the atmosphere.
\end{abstract}

Index terms: Mucuna pruriens var. utilis, green manure, biological nitrogen fixation.

\section{Introdução}

A adubação verde pode ser realizada com espécies de várias famílias botânicas, entre as quais se destacam as da família das leguminosas (Fabaceae). Além de proporcionar benefícios similares aos obtidos com espécies de outras famílias, as leguminosas têm como particularidade o fato de formar associações simbióticas com bactérias dos gêneros Rhizobium e Bradyhizobium, fixadoras de nitrogênio atmosférico. Como resultado da simbiose, quantidades expressivas de nitrogênio $(\mathrm{N})$ tornam-se disponíveis após o corte da leguminosa, o que acarreta auto-suficiência em N, se a planta for adequadamente manejada (Guerra et al., 2004).

Nas regiões tropicais existem inúmeras espécies de leguminosas herbáceas e semiarbustivas, capazes de compor adubo verde, mas, somente umas poucas têm sido estudadas ou são usadas na agricultura (Neves \& Espíndola, 2003). As poucas espécies utilizadas como adubo verde constituem uma base genética estreita e, por isso, seu uso pode acarretar, ao longo do tempo, inconvenientes quanto ao surgimento e susceptibilidade às doenças. 
Para que novas espécies possam ser utilizadas na adubação verde, estudos devem ser conduzidos para avaliar a capacidade de produção de biomassa, a fixação biológica de nitrogênio (FBN), as taxas de cobertura do solo, entre outras características indispensáveis ao adequado manejo da leguminosa.

Entre as espécies de leguminosas com potencial para serem introduzidas nos sistemas agrícolas como adubo verde, destaca-se a mucuna-verde, - Muсuna pruriens (L.) DC. var. utilis (Wall. ex Wight) Baker ex Burck -, pela alta capacidade de produção de biomassa e por não apresentar risco de se tornar uma invasora, já que não apresenta dormência, diferentemente da mucuna-preta [Mucuna aterrima (Piper \& Tracy) Holland] (Brancalião, 2009), que é muito agressiva quando manejada inadequadamente. Contudo, há poucas informações sobre esta espécie, principalmente nas condições ambientais brasileiras.

Além das características intrínsecas a cada espécie de leguminosa utilizada na adubação verde, os aspectos relacionados aos arranjos espaciais interferem na produção de biomassa e na taxa de cobertura do solo (Guerra et al., 2007). Além disso, a taxa de FBN também varia de uma espécie leguminosa para outra (Herridge et al., 2008).

Assim, conhecer o desempenho agronômico de mucuna-verde, em diferentes arranjos espaciais de plantas, contribui para o manejo adequado dessa leguminosa e favorece a diversificação de fontes vegetais para a adubação verde.

O objetivo deste trabalho foi avaliar o efeito de diferentes arranjos espaciais de plantas no desempenho agronômico de mucuna-verde.

\section{Material e Métodos}

O experimento foi instalado no período de dezembro de 2007 a abril de 2008, no campo experimental Terraço da Embrapa Agrobiologia, Município de Seropédica, RJ, $\left(22^{\circ} 48^{\prime} \mathrm{S}, 43^{\circ} 41^{\prime} \mathrm{W}\right.$ e a $33 \mathrm{~m}$ de altitude) em Argissolo Vermelho-Amarelo, cuja análise química na camada de $0-20 \mathrm{~cm}$ apresentou os seguintes resultados: $\mathrm{pH}$ em água $(1: 2,5), 5,2$; $\mathrm{Al}^{+++}, 0,2 \mathrm{cmol}_{\mathrm{c}} \mathrm{dm}^{-3} ; \mathrm{Ca}^{++}, 1,6 \mathrm{cmol}^{\mathrm{c}} \mathrm{dm}^{-3} ; \mathrm{Mg}^{++}$, 0,3 $\mathrm{cmol}_{\mathrm{c}} \mathrm{dm}^{-3} ; \mathrm{P}, 3,3 \mathrm{mg} \mathrm{dm}{ }^{-3} ; \mathrm{K}^{+}, 0,26 \mathrm{cmol}_{\mathrm{c}} \mathrm{dm}^{-3} \mathrm{e}$ $\mathrm{C}$ orgânico, 7,2 $\mathrm{g} \mathrm{kg}^{-1}$. O clima da região é do tipo Aw, segundo a classificação de Köppen e caracteriza-se pela ocorrência de chuvas frequentes e temperaturas muito elevadas, no verão, e pelo inverno pouco rigoroso e geralmente seco. As médias anuais são de cerca de $24^{\circ} \mathrm{C}$ de temperatura e de $1.250 \mathrm{~mm}$ de precipitação pluvial.

Utilizou-se o delineamento de blocos ao acaso, em arranjo fatorial $2 \times 4$, com quatro repetições. Os tratamentos consistiram da semeadura de mucuna-verde, em dois espaçamentos entre sulcos de plantio $(0,5$ e $1,0 \mathrm{~m})$, e quatro densidades de plantas $\left(2,4,8\right.$ e 16 plantas $\left.\mathrm{m}^{-1}\right)$, no total de oito tratamentos. A área total de cada parcela foi de $9,0 \mathrm{~m}^{2}$, com 1,0 $\mathrm{m}^{2}$ central de área útil. A região central foi delimitada por um quadro de $\mathrm{PVC}$, com área interna de $1,0 \mathrm{~m}^{2}$, que continha um referencial de escala colocado sobre o solo no momento da coleta de dados.

A semeadura foi realizada após o preparo do solo com aração, gradagem e sulcamento, tendo-se utilizado altas densidades de plantio, para posterior ajuste da população de plantas, por meio de desbaste manual aos 15 dias após a semeadura. Os tratos culturais consistiram de três capinas manuais, para o controle de plantas espontâneas, realizadas durante o período em que a cultura não havia coberto totalmente o solo.

A taxa de cobertura do solo foi estimada com auxílio do programa SIARCS 3.0 (Jorge \& Crestana, 1996). O acompanhamento do crescimento das plantas, até a plena cobertura do solo, foi feito por meio de imagens fotográficas, com resolução de 3 mega pixels, tomadas da área útil aos 18, 30, 36, 40, 57 e 70 dias após a semeadura (DAS). A câmera fotográfica digital era suspensa de modo que as fotografias fossem tomadas perpendicularmente ao solo, à altura de $1,80 \mathrm{~m}$ da superfície. Cada fotografia abrangia uma área de $1 \mathrm{~m}^{2}$, e foi tomada entre 7 e $9 \mathrm{~h}$ da manhã, a fim de atenuar efeitos adversos da sombra. Um editor foi utilizado para fazer o corte das imagens, a fim de se deixar apenas a área útil para posterior processamento (separação da área coberta da área desnuda) e obtenção, com base no referencial de escala, da percentagem de cobertura do solo no programa SIARCS.

Para a avaliação da produção de biomassa da parte aérea, o corte foi feito rente à superfície do solo, aos 140 DAS, quando as plantas tinham aproximadamente $50 \%$ das flores abertas. A massa de matéria fresca da parte aérea foi pesada em balança com capacidade para $6,0 \mathrm{~kg}$. Após a pesagem, as subamostras dos tratamentos foram retiradas, acondicionadas em sacos de papel e transferidas para estufa de circulação forçada de ar, a $65^{\circ} \mathrm{C}$, até alcançar massa constante. 
Após a secagem, as amostras foram processadas em moinho tipo Wiley, com abertura de malha de 20 mesh. Em seguida, foram retiradas amostras para a determinação da matéria seca, do acúmulo de $\mathrm{N}$ total, pelo método de Kjeldahl, conforme Alves et al. (1994) e da taxa de FBN, pela técnica de abundância natural do isótopo ${ }^{15} \mathrm{~N}$, conforme Ramos et al. (2001). As espécies de ocorrência espontânea e não leguminosas, capim-colonião (Panicum maximum), emília (Emilia sonchifolia) e pé-de-galinha (Eleusine indica) foram também coletadas das proximidades da área experimental, secas a $65^{\circ} \mathrm{C}$, moídas e analisadas quanto à abundância natural do isótopo ${ }^{15} \mathrm{~N}$ (Okito et al., 2004a), para serem utilizadas como referência da abundância natural do isótopo ${ }^{15} \mathrm{~N}$ do $\mathrm{N}$ disponível no solo.

A percentagem de $\mathrm{N}$ derivada da $\mathrm{FBN}$ na planta $(\% \mathrm{FBN})$ foi calculada pela equação,

$$
\% \mathrm{FBN}=\left(\delta^{15} \mathrm{Nc}-\delta^{15} \mathrm{Nm}\right) /\left(\delta^{15} \mathrm{Nc}-\mathrm{B}\right) 100,
$$

em que: $\delta^{15} \mathrm{Nc}$ e $\delta^{15} \mathrm{Nm}$ correspondem aos valores de abundância natural de ${ }^{15} \mathrm{~N}$ das plantas controle e da mucuna-verde, respectivamente; e B é uma constante relacionada ao processo de discriminação isotópica de ${ }^{15} \mathrm{~N}$, pelo processo da $\mathrm{FBN}$, considerada no presente trabalho como -1,54 delta (Okito et al., 2004a). Em todos os tratamentos, a quantidade total de $\mathrm{N}$ na planta, derivada da FBN, foi calculada pela multiplicação da \%FBN pelo total de $\mathrm{N}$ acumulado pela planta.

As análises estatísticas foram realizadas com auxílio dos programas SAEG, versão 5.0 (Euclydes, 1983) e SISVAR, versão 5.3 (Ferreira, 2008), e consistiram da análise de variância, seguida da escolha, pelo aplicativo Sigma Plot, versão 11, do modelo com melhor ajuste das curvas; e aplicação do teste $\mathrm{F}$ aos coeficientes da equação, a $5 \%$ de probabilidade. Quanto à taxa de cobertura do solo, as curvas foram ajustadas de acordo com a função sigmoidal de três parâmetros: $\mathrm{TC}=\mathrm{TC}_{\text {máx }} /\left\{1+\exp \left[-\left(\mathrm{DAS}-\mathrm{t}_{1 / 2}\right) / \mathrm{B}\right]\right\}$, em que: TC é a cobertura do solo, em percentagem, no tempo DAS, em

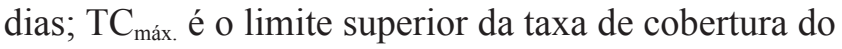
solo; $\mathrm{t}_{1 / 2}$ é a idade da mucuna-verde, em dias, quando alcançou $50 \%$ de cobertura de terreno; B é o período de tempo, em dias, de incremento máximo em torno de $t_{1 / 2}$. Em relação à produção de matéria seca da parte aérea e acúmulo total de $\mathrm{N}$, a escolha do modelo para representar a distribuição dos dados foi baseada no comportamento biológico, a 5\% de probabilidade, e no coeficiente de determinação $\left(\mathrm{R}^{2}\right)$.

\section{Resultados e Discussão}

Verificou-se efeito significativo $(\mathrm{p} \leq 0,05)$ do espaçamento entre sulcos de plantio e da densidade de plantas, na taxa de cobertura do solo, e não houve interação entre esses fatores.

A mucuna-verde cobriu totalmente o solo, aos 70 DAS, nos dois espaçamentos entre sulcos (Figura 1). Para mucuna-cinza [Mucuna cinereum (Piper \& Tracy)], cultivada em população de 20 plantas $\mathrm{m}^{-2}$, esse período corresponde a 37 dias (Guerra et al., 2007). Assim, a mucuna-verde apresenta velocidade de cobertura do solo relativamente inferior à de mucuna-cinza. Deve-se destacar que, durante a fase de crescimento exponencial ou intervalo de incremento máximo em torno do $\mathrm{t}_{1 / 2}$ (idade, em dias, quando alcançou $50 \%$ de cobertura de terreno), a mucuna-verde aumentou a cobertura do solo de 25 para $75 \%$, em intervalo de 15 e de 19 dias, respectivamente, para os espaçamentos de $0,5 \mathrm{~m}$ e de $1,0 \mathrm{~m}$ entre sulcos de plantio.

Observou-se que na densidade de quatro plantas por metro e espaçamento entre sulcos de $0,5 \mathrm{~m}$, densidade que é normalmente utilizada para mucuna-cinza e mucuna-preta (Nascimento et al., 2005), o intervalo de tempo necessário, para que metade da superfície

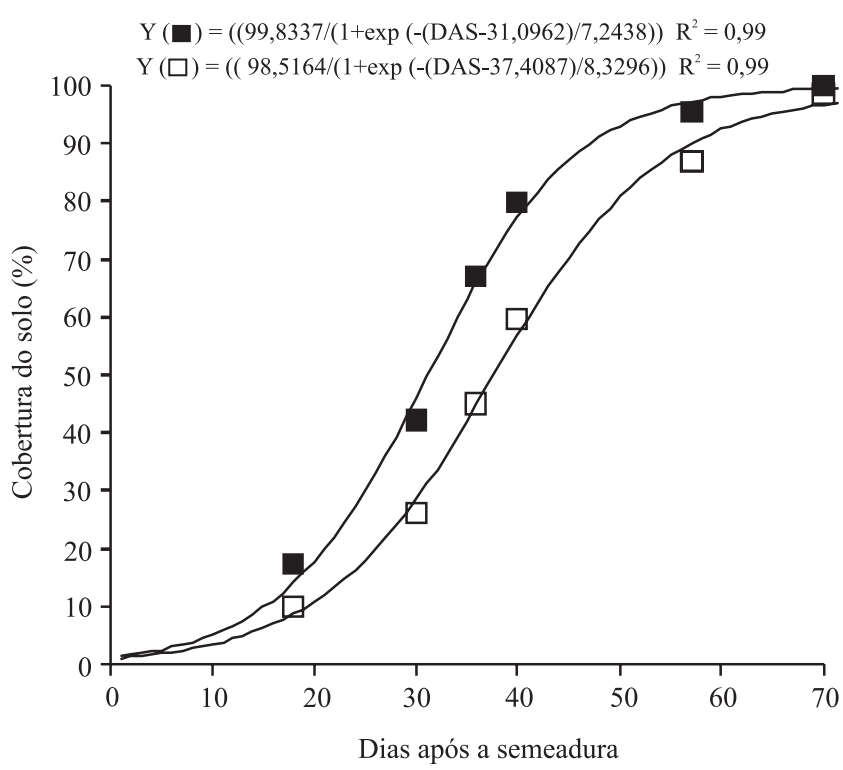

Figura 1. Cobertura do solo por mucuna-verde, em função de espaçamento entre sulcos de 0,5 m (ם) e 1,0 m ( $\square$ ) e de dias após a semeadura. 
do solo estivesse coberta $\left(t_{1 / 2}\right)$, foi de 34 DAS. Esse tempo foi reduzido para 24 DAS, quando a densidade foi de 16 plantas por metro, nesse mesmo espaçamento (Figura 2). Todavia, levando-se em conta a maior necessidade de sementes na densidade de 16 plantas por metro e o respectivo custo referente à aquisição delas, essa diferença de tempo deve ser relativizada, pois populações menores cumprem papel semelhante. Por exemplo, é possível aumentar o espaçamento entre sulcos para $1 \mathrm{~m}$ e manter a densidade de quatro plantas por metro, aumentando-se o $\mathrm{t}_{1 / 2} \mathrm{em}$ apenas seis dias.

Esses resultados corroboram os obtidos por Sodré Filho et al. (2004) para mucuna-preta. Esses autores observaram que, aos $30 \mathrm{DAS}$, a mucuna-preta apresenta taxa de cobertura de $39 \%$ e, aos $60 \mathrm{DAS}$, esse valor é de $60 \%$. De acordo com os resultados obtidos no presente trabalho, a mucuna-verde apresentou bom potencial para cobrir a superfície do solo já entre o segundo e o terceiro mês de cultivo.

O espaçamento, a densidade e a interação entre esses fatores não influenciaram a taxa de FBN (Tabela 1). A estimativa do $\mathrm{N}$ derivado da FBN na mucuna-verde

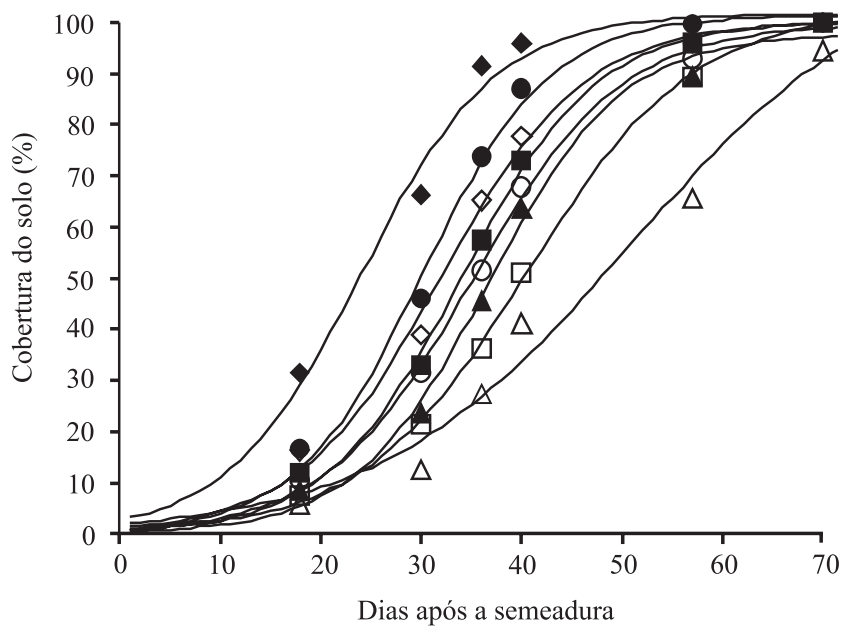

Figura 2. Cobertura do solo por mucuna-verde, em função das densidades de $2(\triangle), 4(\square), 8(\circ)$ e $16(\diamond)$ plantas $\mathrm{m}^{-1}$ e de dias após a semeadura, no espaçamento de 1,0 $\mathrm{m}$ entre sulcos de plantio, e de $2(\boldsymbol{\Delta}), 4(\boldsymbol{\bullet}), 8(\bullet)$ e $16(\diamond)$ plantas $\mathrm{m}^{-1}$, no espaçamento de $0,5 \mathrm{~m}$ entre sulcos de plantio . foi, em média, de 67\%. Esta taxa encontra-se dentro da faixa de FBN realizada por leguminosas utilizadas nos sistemas agrícolas, que é de 40 a $70 \%$, conforme Herridge et al. (2008). Okito et al. (2004b) observaram que a contribuição da FBN para mucuna-preta (Mucuna aterrima), cultivada nas mesmas condições edafoclimáticas do presente trabalho, foi de $69 \%$, similar ao valor observado para mucuna-verde.

No espaçamento de $1 \mathrm{~m}$ entre sulcos de plantio, o aumento do número de plantas por metro ocasionou aumento significativo na quantidade de $\mathrm{N}$ derivado da FBN (N-FBN) (Tabela 1). A quantidade de N-FBN variou de 53,55 a $93,64 \mathrm{~kg} \mathrm{ha}^{-1}$ de $\mathrm{N}$ e foi o melhor ajuste obtido pelo modelo linear. No espaçamento de $0,5 \mathrm{~m}$ entre sulcos, o N-FBN variou de 60,63 a $65,71 \mathrm{~kg} \mathrm{ha}^{-1}$ de $\mathrm{N}$, e não foram observadas diferenças significativas na quantidade de N-FBN em função da densidade de plantas.

A quantidade do N-FBN variou em função da densidade da mucuna-verde, e a maior quantidade de N-FBN foi obtida no espaçamento de $1 \mathrm{~m}$ entre sulcos e 16 plantas por metro. Esses resultados mostram que, em uma situação na qual o valor da semente não é fator limitante, pode-se utilizar essa densidade. Entretanto, a utilização de apenas quatro plantas por metro, no espaçamento de $1 \mathrm{~m}$, é capaz de proporcionar acúmulo de mais de $50 \mathrm{~kg} \mathrm{ha}^{-1}$ de $\mathrm{N}$, derivado da FBN. Esse resultado torna-se ainda mais relevante, ao se considerar que esse espaçamento, por ser mais aberto, favorece a introdução de espécies de importância econômica entre os sulcos da mucuna-verde. Essa quantidade de N-FBN é similar à obtida por Okito et al. (2004b) para

Tabela 1. Taxa de fixação biológica de nitrogênio (FBN) e nitrogênio derivado da FBN (N-FBN) acumulado na parte aérea da mucuna-verde, em função de diferentes espaçamentos entre sulcos $(0,5$ e $1,0 \mathrm{~m})$ e densidades de plantas por metro, aos 70 dias após a semeadura ${ }^{(1)}$.

\begin{tabular}{lcccc}
\hline Densidade $\left(\right.$ planta $\mathrm{m}^{-1}$ ) & \multicolumn{2}{c}{$0,5 \mathrm{~m}$} & \multicolumn{2}{c}{$1,0 \mathrm{~m}$} \\
\cline { 2 - 5 } & FBN (\%) & N-FBN & FBN (\%) & N-FBN* \\
\hline 2 & 72,9 & 59,82 & 63,9 & 56,32 \\
4 & 65,0 & 65,71 & 63,2 & 53,55 \\
8 & 69,1 & 55,63 & 66,8 & 69,34 \\
16 & 70,3 & 59,94 & 70,7 & 93,64 \\
\hline
\end{tabular}

${ }^{(1)} \mathrm{O}$ valor de $\delta 15 \mathrm{~N}(\%)$ das plantas de mucuna-verde foi de $0,71 \pm 0,10$ e os das plantas utilizadas como controle foram: 4,63 $\pm 0,06$ para capimcolonião (Panicum maximum); 5,71 $\pm 0,23$ para emília (Emilia sonchifolia) e $6,16 \pm 0,19$ para pé-de-galinha (Eleusine indica); $*$ Significativo a $5 \%$ de probabilidade, pelo teste de $\mathrm{F} . \mathrm{y}=12,775 \mathrm{x}+36,27(\mathrm{R} 2=0,81)$. 
mucuna-preta, nas mesmas condições edafoclimáticas do presente trabalho.

Houve interação significativa dos fatores espaçamento entre sulcos e o número de plantas por metro para produção de matéria seca e quantidade de $\mathrm{N}$ total acumulado na parte aérea (Figura 3). A produção de matéria seca apresentou comportamento linear e aumentou significativamente, à medida em que a densidade de plantas também aumentava, no espaçamento de $1,0 \mathrm{~m}$ entre sulcos. No espaçamento de $0,5 \mathrm{~m}$ entre sulcos, não foram observadas alterações no comportamento da mucuna-verde em função da densidade de plantas. Isso mostra que, ao se utilizar o espaçamento entre sulcos de 0,5 m, deve-se optar pela densidade de quatro plantas por metro, pois, nessa condição não há incremento na produção de matéria seca com o aumento do número de plantas por metro. No espaçamento entre sulcos de $1 \mathrm{~m}$, o aumento do número de plantas por metro resulta em ganho
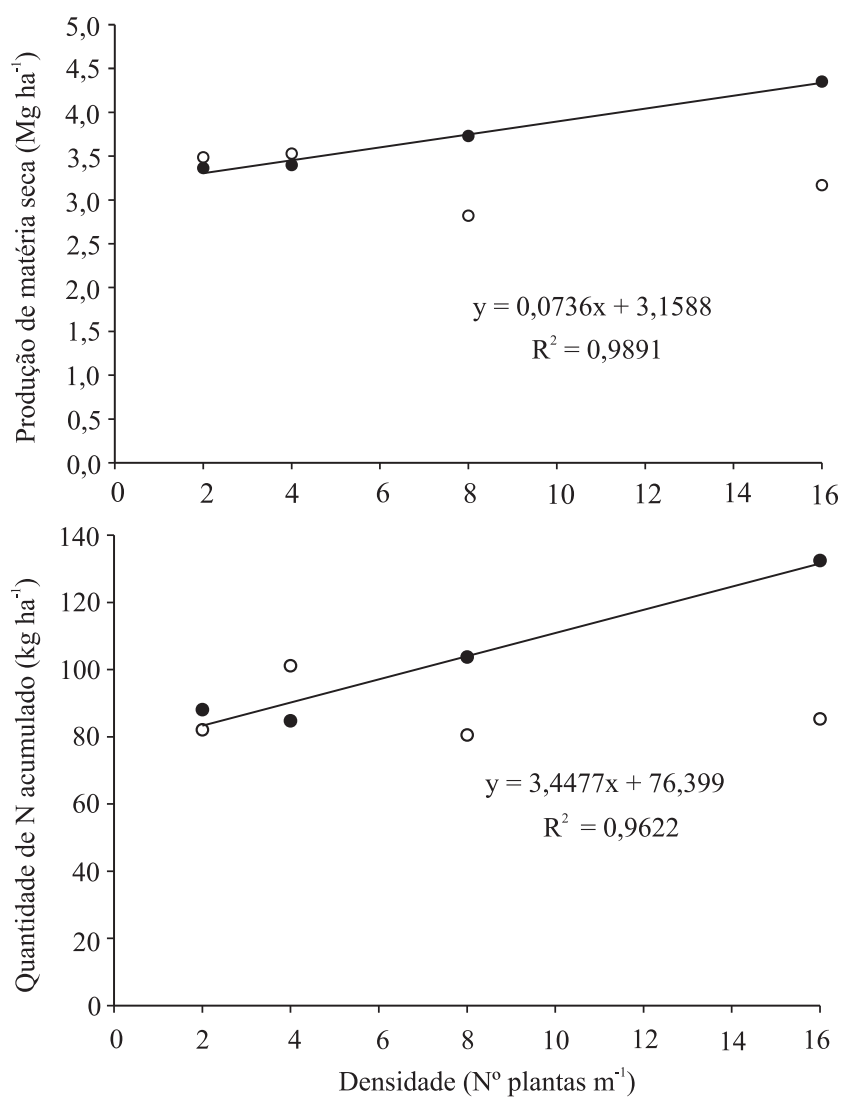

Figura 3. Produtividade de matéria seca e quantidade de nitrogênio acumulado na parte aérea de mucuna-verde, em função das densidades de 2, 4, 8 e 16 plantas $\mathrm{m}^{-1}$, nos espaçamentos de $0,5 \mathrm{~m}(\circ)$ e $1,0 \mathrm{~m}(\bullet)$ entre sulcos de plantio. na produção de matéria seca. Nesse espaçamento, a produtividade máxima é atingida com o uso de 16 plantas por metro. Nessa densidade, a produtividade de matéria seca foi de 4,7 $\mathrm{Mg} \mathrm{ha}^{-1}$, similar aos resultados obtidos por Carvalho et al. (2004) para mucuna-preta.

A quantidade de $\mathrm{N}$ total acumulado na parte aérea da mucuna-verde seguiu a mesma tendência observada para a produção de matéria seca. No espaçamento de $1,0 \mathrm{~m}$ entre sulcos, houve incremento significativo com o aumento do número de plantas por metro (Figura 3). Este comportamento não foi observado para o espaçamento de $0,5 \mathrm{~m}$ entre sulcos de plantio. A densidade de 16 plantas por metro no espaçamento de 1,0 m entre sulcos de plantio, proporciona maior produção de matéria seca e nitrogênio. Esse tratamento resultou em acúmulo de cerca de $100 \mathrm{~kg} \mathrm{ha}^{-1}$ de $\mathrm{N}$ na ocasião da colheita, valor que é cerca de quatro vezes superior ao observado por Oliveira et al. (2002) para mucuna-preta.

\section{Conclusões}

1. O arranjo espacial de 16 plantas por metro, no espaçamento de $1,0 \mathrm{~m}$ entre sulcos de plantio, proporciona maior produção de matéria seca e acúmulo de $\mathrm{N}$ na parte aérea de mucuna-verde.

2. Para a cobertura do solo, o arranjo espacial mais adequado é de 16 plantas por metro, no espaçamento de $0,5 \mathrm{~m}$ entre sulcos de plantio.

3. O uso de apenas quatro plantas por metro, no espaçamento de $1 \mathrm{~m}$, proporciona acúmulo de mais de $50 \mathrm{~kg} \mathrm{ha}^{-1}$ de $\mathrm{N}$ derivado do processo de fixação biológica de nitrogênio.

\section{Agradecimentos}

À Fundação de Apoio à Pesquisa do Estado do Rio de Janeiro, ao Conselho Nacional de Desenvolvimento Científico e Tecnológico e à Embrapa, pelo apoio financeiro.

\section{Referências}

ALVES, B.J.R.; SANTOS, J.C.F. dos; URQUIAGA, S.; BODDEY, R.M. Métodos de determinação do nitrogênio em solo e planta. In: HUNGRIA, M.; ARAUJO, R.S. (Ed.). Manual de métodos empregados em estudos de microbiologia agrícola. Brasília: Embrapa SPI, 1994. p.449-470. (Embrapa-CNPAF. Documentos, 46).

BRANCALIÃO, S.R. Viabilidade do uso de "coquetel" comparado a leguminosas solteiras em sucessão com a cultura 
da soja no sistema plantio direto em Campinas (SP). 2009. Disponível em: <http://www.infobibos.com/Artigos/2009_1/ coquetel/index.htm>. Acesso em: 27 abr. 2010.

CARVALHO, M.A.C. de; ATHAYDE, M.L.F.; SORATTO, R.P.; ALVES, M.C.; SÁ, M.E. de. Adubação verde e sistemas de manejo do solo na produtividade do algodoeiro. Pesquisa Agropecuária Brasileira, v.39, p.1205-1211, 2004.

EUCLYDES, R.F. SAEG: sistema para análises estatísticas e genéticas. Versão 5.0. Viçosa: UFV, 1983.

FERREIRA, D.F. SISVAR: um programa para análises e ensino de estatística. Revista Symposium, v.6, p.36-41, 2008.

GUERRA, J.G.M.; DE-POLLI, H.; ALMEIDA, D.L. de. Managing carbon and nitrogen in tropical organic farming through green manuring. In: ADETOLA BADEJO, M.; TOGUN, A.O. (Org.). Strategies and tactics of sustainable agriculture in the tropics. Ibadan: College Press, 2004. p.125-140.

GUERRA, J.G.M.; ESPÍNDOLA, J.A.A.; PERIN, A.; TEIXEIRA, M.G.; ALMEIDA, D.L. de; ASSIS, R.L. de. Desempenho de leguminosas tropicais perenes como plantas de cobertura do solo. Seropédica: Embrapa Agrobiologia, 2007. 39p. (Embrapa Agrobiologia. Boletim de pesquisa e desenvolvimento, 20).

HERRIDGE, D.F.; PEOPLES, M.B.; BODDEY, R.M. Global inputs of biological nitrogen fixation in agricultural systems. Plant and Soil, v.311, p.1-18, 2008.

JORGE, L.A.C.; CRESTANA, S. SIARCS 3.0: novo aplicativo para análise de imagens digitais aplicado à ciência do solo. In:
CONGRESSO LATINO-AMERICANO DE CIÊNCIA DO SOLO, 13., 1996, Águas de Lindóia. Anais. Campinas: Sociedade Brasileira de Ciência do Solo, 1996. 5p.

NASCIMENTO, J.T.; SILVA, I. de F. da; SANTIAGO, R.D.; SILVA NETO, L. de F. da. Efeito de leguminosas nos atributos físicos e carbono orgânico de um Luvissolo. Revista Brasileira de Ciência do Solo, v.29, p.825-831, 2005.

NEVES, M.C.P.; ESPÍNDOLA, J.A.A. A adubação verde e as bactérias do solo. Agroecologia Hoje, n.22, p.4-5, 2003.

OKITO, A.; ALVES, B.J.R.; URQUIAGA, S.; BODDEY, R.M. Isotopic fractionation during $\mathrm{N}_{2}$ fixation by four tropical legumes. Soil Biology and Biochemistry, v.36, p.1179-1190, 2004a.

OKITO, A.; ALVES, B.J.R.; URQUIAGA, S.; BODDEY, R.M. Nitrogen fixation by groundnut and velvet bean and residual benefit to a subsequent maize crop. Pesquisa Agropecuária Brasileira, v.39, p.1183-1190, 2004b.

OLIVEIRA, T.K. de; CARVALHO, G.J. de; MORAES, R.N. de S. Plantas de cobertura e seus efeitos sobre o feijoeiro em plantio direto. Pesquisa Agropecuária Brasileira, v.37, p.1079-1087, 2002.

RAMOS, M.G.; VILLATORO, M.A.A.; URQUIAGA, S.; ALVES, B.J.R.; BODDEY, R.M. Quantification of the contribution of biological nitrogen fixation to tropical green manure crops and the residual benefit to a subsequent maize crop using ${ }^{15} \mathrm{~N}$-isotope techniques. Journal of Biotechnology, v.91. p.105-115, 2001.

SODRÉ FILHO, J.S.; CARDOSO, A.N.; CARMONA, R.; CARVALHO, A.M. de. Fitomassa e cobertura do solo de culturas de sucessão ao milho na Região do Cerrado. Pesquisa Agropecuária Brasileira, v.39, p.327-334, 2004.

Recebido em 30 de dezembro de 2010 e aprovado em 4 de maio de 2011 